hep-th/9611176

EFI-96-40

YCTP-P22-96

\title{
Exact Gravitational Threshold Correction in the FHSV model
}

\author{
Jeffrey A. Harvey \\ Enrico Fermi Institute, University of Chicago \\ 5640 Ellis Avenue, Chicago, IL 60637 \\ harvey@poincare.uchicago.edu \\ Gregory Moore \\ Department of Physics, Yale University \\ New Haven, CT 06511 \\ moore@castalia.physics.yale.edu
}

\begin{abstract}
We consider the automorphic forms which govern the gravitational threshold correction $F_{1}$ in models of heterotic/IIA duality with $N=2$ supersymmetry in four dimensions. In particular we derive the full nonperturbative formula for $F_{1}$ for the dual pair originally considered by Ferrara, Harvey, Strominger and Vafa (FHSV). The answer involves an interesting automorphic product constructed by Borcherds which is associated to the "fake Monster Lie superalgebra." As an application of this result we rederive a result of Jorgenson \& Todorov on determinants of $\bar{\partial}$ operators on $K 3$ surfaces.
\end{abstract}

Nov. 18, 1996 


\section{Introduction}

In previous work we have developed the idea that there is an algebraic structure which underlies many dualities in string theory [1,2]. This point of view is supported by the fact that threshold corrections in $N=2$ theories are given by sums of logarithmic functions over the roots of generalized Kac Moody algebras (GKM) [1] and by the fact that one can define an algebra on BPS states which in some cases is closely related to a generalized Kac-Moody algebra [2]. Since threshold corrections are determined purely by the spectrum of BPS states, there must be a relation between these two facts. Unfortunately the precise relation is still not clear. In fact, to our knowledge the denominator product of a known GKM has not yet appeared in a calculation of threshold corrections in any model (such products have appeared as part of the answer in several papers). In this paper we will remedy this by showing that the denominator function of the "fake Monster Lie superalgebra" [3] governs the gravitational threshold correction $F_{1}$ in the $N=2$ dual pair of FHSV [4].

In the second and third sections of this paper we discuss some general features of gravitational threshold corrections in $N=2$ heterotic and type IIA dual pairs. In the fourth section we apply these results and second quantized mirror symmetry to determine the exact non-perturbative form for $F_{1}$ in the FHSV dual pair. The fifth section applies

this result to rederive a result of Jorgenson and Todorov on the determinant of $\bar{\partial}$ operators on $K 3$ surfaces which double cover the Enriques surface. We make a few brief comments on the relation to $\mathrm{F}$ theory in section 6 and conclude in section 7 .

\section{Gravitational threshold correction for $d=4, \mathcal{N}=2$ heterotic compactifica- tions}

\subsection{One-loop integral}

We first consider $d=4, N=2$ heterotic compactifications with the ten-dimensional gauge group broken to some rank $s$ subgroup (there are a total of $s+4$ vector gauge fields). The tree level vectormultiplet moduli space is therefore:

$$
\begin{aligned}
\mathcal{M}_{v m} & =\left\{\left(\tau_{S}, y\right)\right\} \in S L(2, \mathbb{R}) / S O(2) \times \mathcal{N}^{s+2,2} \\
\mathcal{N}^{s+2,2} & =O\left(\Gamma^{s+2,2}\right) \backslash O(s+2,2) /[O(s+2) \times O(2)] .
\end{aligned}
$$

String and automorphic fields $S, \tau_{S}$, respectively are defined as follows. We choose $\operatorname{Re}(S)>$ 0 and define

$$
q_{S} \equiv e^{-8 \pi^{2} S}=e^{2 \pi i \tau_{S}}
$$


which is invariant under axion shifts. We normalize the Yang-Mills fields so that the action is $\frac{1}{2 g^{2}} \int \operatorname{tr} F \wedge * F$ with $S=\frac{1}{g^{2}}+i \frac{\theta}{8 \pi^{2}}$.

In this paper we focus on the effective, non-Wilsonian coupling

$$
\int_{\mathbb{R}^{1,3}} \frac{1}{24 g_{\text {grav }}^{2}\left(p^{2}\right)} \operatorname{tr} R \wedge R^{*}
$$

At string tree level $g_{\text {grav }}^{-2}\left(p^{2}\right)=24 R e(S)$. These couplings have been widely discussed in the literature [5, 6, 7, 8, 9, 10, 11]. A one-loop formula for the derivatives of $g_{\text {grav }}$ with respect to the vector moduli was derived in [5]:

$$
\frac{\partial}{\partial y^{i}}\left(\frac{1}{g_{\text {grav }}^{2}\left(p^{2}\right)}\right)=\frac{\partial}{\partial y^{i}} \frac{\Delta_{\text {grav }}^{(1)}}{16 \pi^{2}}
$$

where in the above we are holding the string coupling $S$ fixed. The quantity $\Delta_{\text {grav }}^{(1)}$ is given in [5] as

$$
\Delta_{\text {grav }}^{(1)}=\int_{\mathcal{F}} \frac{d^{2} \tau}{\tau_{2}}\left[\frac{-i}{\eta^{2}(\tau)} \operatorname{Tr}_{R}\left\{J_{0} e^{i \pi J_{0}} q^{L_{0}-c / 24} \bar{q}^{\bar{L}_{0}-\bar{c} / 24}\left(E_{2}(\tau)-\frac{3}{\pi \tau_{2}}\right)\right\}-b_{\text {grav }}\right]
$$

where the trace is over the Ramond sector, $J_{0}$ is the $U(1)$ current of the $N=2$ superconformal algebra, and $E_{2}$ is the second Eisenstein series with a $q$ expansion

$$
E_{2}=1-24 \sum_{n=1}^{\infty} \sigma(n) q^{n}
$$

with $\sigma(n)$ the sum of the divisors of $n$. The constant $b_{\text {grav }}$ is the "gravitational beta function" of [5] and ensures that the integral (2.5) is finite away from enhanced symmetry subvarieties. From (2.5) we may obtain the duality invariant effective coupling:

$$
\left(\frac{1}{g_{\text {grav }}^{2}\left(p^{2}\right)}\right)^{H E T}=\frac{24}{g_{\text {string }}^{2}}+\frac{b_{\text {grav }}}{16 \pi^{2}} \log \frac{M_{\text {Planck }}^{2}}{p^{2}}+\frac{\Delta_{\text {grav }}^{(1)}}{16 \pi^{2}}-\frac{5 n_{V}+n_{H}}{8 \pi^{2}} \log [R e S]
$$

where $g_{\text {string }}^{-2}=\operatorname{Re}(S)+\Delta^{\text {univ }} / 16 \pi^{2}, n_{H}$ is the number of massless hypermultiplets, and $n_{V}$ is the number of massless vectormultiplets (including the graviphoton). The quantity $\Delta^{\text {univ }}$ is related to a Green-Schwarz term for sigma-model anomalies. We have chosen to write the second term in (2.7) with the Planck mass rather than the string scale in the logarithm since it is the Planck mass that is duality invariant. Actually, (2.7) does not quite follow from (2.4). The last term in (2.7), which affects only the dilaton one-point 
function, is very difficult to compute and is not determined by (2.4). 1 It may however be determined by appealing to heterotic-IIA string duality. In particular it can be determined from the expressions we derive in section 3 for the corresponding quantities in IIA theory.

Now we derive two important properties of the integral (2.5). From [1] we have the formula:

$$
\begin{gathered}
\frac{i}{2} \frac{1}{\eta^{2}} \operatorname{Tr}_{\mathrm{R}} \mathrm{J}_{0} \mathrm{e}^{\mathrm{i} \pi \mathrm{J}_{0}} \mathrm{q}^{\mathrm{L}_{0}-\mathrm{c} / 24} \bar{q}^{\tilde{L}_{0}-\tilde{c} / 24}= \\
\left.\sum_{\text {BPS vectormultiplets }} q^{\Delta} \bar{q}^{\bar{\Delta}}-\sum_{\text {BPS hypermultiplets }} q^{\Delta} \bar{q}^{\bar{\Delta}}\right]
\end{gathered}
$$

This implies:

1. Using the expansion (2.6) and noting that (2.8) starts with $q^{-1}+\cdots$ we see that the constant is

$$
b_{\text {grav }}=2\left(n_{H}-n_{V}+24\right)
$$

thus recovering the result of [5].

2. If we approach an enhanced symmetry variety at which masses of BPS vector multiplets, $m_{v}$, and/or masses of BPS hypermultiplets, $m_{h}$ approach zero then the integral (2.5) picks up a divergence

$$
2\left[\sum_{\text {BPS vectormultiplets }} \log \left(4 \pi m_{v}^{2}\right)-\sum_{\text {BPS hypermultiplets }} \log \left(4 \pi m_{h}^{2}\right)\right]
$$

\subsection{Evaluating the integral}

Using the techniques of [12,1] the above integral can be evaluated for "rational backgrounds" which satisfy:

$$
\frac{i}{2} \frac{1}{\eta^{2}} \operatorname{Tr}_{\mathrm{R}} \mathrm{J}_{0} \mathrm{e}^{\mathrm{i} \pi \mathrm{J}_{0}} \mathrm{q}^{\mathrm{H}} \overline{\mathrm{q}}^{\tilde{\mathrm{H}}}=\sum_{\mathrm{i}} \mathrm{Z}_{\Gamma}^{\mathrm{i}}(\mathrm{q}, \overline{\mathrm{q}}) \mathrm{f}_{\mathrm{i}}(\mathrm{q})
$$

where $f_{i}(q)$ form a unitary representation of $S L(2, \mathbb{Z})$ and $Z_{i}$ are a set of partition functions for a lattice and its translates.

The evaluation of (2.5) was done in [1, 13, 14, 15,] with the following result. Introduce the pseudo-invariant dilaton:

$$
\tilde{S}=S+\frac{1}{s+4} \eta^{a b} \frac{\partial}{\partial y^{a}} \frac{\partial}{\partial y^{b}} h^{(1)}
$$

1 Deriving the last term in (2.7) from a low energy field theory analysis is an extremely challenging problem. We thank V. Kaplunovsky and J. Louis for detailed discussions about this. 
such that $\operatorname{Re}(\tilde{S})$ is invariant under $T$-duality. In the above $h^{(1)}$ is the one-loop prepotential. Then we have at 1-loop: 2

$$
\frac{4 \pi^{2}}{3}\left(\frac{1}{g_{\text {grav }}^{2}\left(p^{2}\right)}\right)^{H E T}=\frac{b_{\text {grav }}}{12} \log \frac{M_{\text {Planck }}^{2}}{p^{2}}-\log \left\|\Psi_{\text {grav }}^{\leq 1}(S, y)\right\|^{2}
$$

where

$$
\begin{gathered}
\log \left\|\Psi_{\text {grav }}^{\leq 1}(S, y)\right\|^{2}=-4 R e\left[8 \pi^{2} \tilde{S}\right]+\frac{5 n_{V}+n_{H}}{6} \log [\operatorname{Re} S]+\log \left\|\Psi_{\text {grav }}(y)\right\|^{2} \\
\log \left\|\Psi_{\text {grav }}(y)\right\|^{2}=\log \left|\Psi_{\text {grav }}(y)\right|^{2}+\frac{b_{\text {grav }}}{12}\left(\log \left[-(\text { Imy })^{2}\right]-\mathcal{K}\right)
\end{gathered}
$$

and

$$
\Psi_{\text {grav }}^{\leq 1}(S, y)=q_{\tilde{S}}^{2} \Psi_{\text {grav }}(y)
$$

The quantity $\mathcal{K}$ in (2.14) is an unimportant constant defined in [1]. Each term on the RHS of (2.13) is $T$-duality invariant. $T$-duality invariance and (2.10) lead to the following key properties of $\Psi_{\text {grav }}(y)$ :

1. $\Psi_{\text {grav }}(y)$ is a modular form of $O(s+2,2 ; \mathbb{Z})$ of weight

$$
w=\frac{b_{\text {grav }}}{12}=4-\frac{n_{V}-n_{H}}{6} .
$$

2. If $y$ approaches a subvariety where there are new massless vector and hyper multiplets then the LHS of (2.13) diverges like:

$$
\frac{1}{6}\left[\sum_{v m} \log \left(4 \pi m^{2}\right)-\sum_{h m} \log \left(4 \pi m^{2}\right)\right]
$$

Therefore, if $\tilde{S}$ does not pick up a divergence, and if there are $\Delta n_{V}$ vector multiplets and $\Delta n_{H}$ hypermultiplets with masses vanishing to first order in the distance to the subvariety then $\Psi_{\text {grav }}(y)$ obtains a zero of order:

$$
\frac{1}{6}\left[\Delta n_{H}-\Delta n_{V}\right]
$$

\subsection{Loop corrections}

Finally, let us discuss higher loops. The above result (2.13) can be cast in the form:

$$
\begin{aligned}
\left(\frac{1}{g_{\text {grav }}^{2}\left(p^{2}\right)}\right)^{H E T} & =\operatorname{Re}\left[\mathcal{F}_{1}^{H E T}(S, y)\right]+\frac{b_{\text {grav }}}{16 \pi^{2}}\left[\log \frac{M_{\text {Planck }}^{2}}{p^{2}}+K^{(0)}(S, \bar{S}, y, \bar{y})\right] \\
& -\frac{3}{4 \pi^{2}}\left(4-n_{V}\right) \log [\operatorname{Re} S]
\end{aligned}
$$

2 The strange prefactor is meant to define a nice modular form. It also facilitates the comparison with the IIA string. 
where $K^{(0)}$ is the tree level Kahler potential, $K^{(0)}=-\log R e S-\log \left[-(\operatorname{Im} y)^{2}\right] . \mathcal{F}_{1}^{H E T}(S, y)$ is holomorphic, and has transformation properties such that $g_{\text {grav }}^{-2}\left(p^{2}\right)$ is invariant. As discussed in [9], $N=2$ supersymmetry says that

$$
\mathcal{F}_{1}^{H E T}(S, y)=24 S+\mathcal{F}_{1}^{H E T,(1)}(y)+\mathcal{O}\left(e^{-8 \pi^{2} S}\right)
$$

so (2.13) implies the formula for $\mathcal{F}_{1}^{H E T}(S, y)$ to all orders of perturbation theory. Moreover, as the notation suggests, we expect that $\Psi_{\text {grav }}^{\leq 1}(S, y)$ in 2.15 should be regarded as the first term of an expansion in $q_{S}$ of an automorphic product on the exact vectormultiplet moduli space. Thus, it should be possible to understand the $\log [\operatorname{Re} S]$ correction purely within the heterotic string by requiring $T$-duality invariance of the invariant norm-square $\|\Psi(S, y)\|^{2}$ of the full product. However, this might require a nonperturbative understanding of certain infrared effects.

\section{Gravitational threshold correction for $d=4, \mathcal{N}=2$ type II compactification}

We now turn to gravitational threshold corrections in type II string theory on a CalabiYau 3-fold $X_{3}$ with Kahler potential $K$, Betti numbers $h^{1,1}$ and $h^{2,1}$ and Euler number $\chi=2\left(h^{1,1}-h^{2,1}\right)$.

The Gauss-Bonnet coupling for the type II string on a Calabi-Yau 3-fold $X_{3}$ is given by [16,8]:

$$
\frac{4 \pi^{2}}{3}\left(\frac{1}{g_{\text {grav }}^{2}\left(p^{2}\right)}\right)^{I I}=\frac{b_{\text {grav }}}{12} \log \frac{M_{\text {Planck }}^{2}}{p^{2}}+F_{1}^{k a h l e r}
$$

where the last term is obtained from a fundamental domain integral:

$$
\begin{aligned}
F_{1} & =\int_{\mathcal{F}} \frac{d^{2} \tau}{\tau_{2}}\left[\operatorname{Tr}_{\mathrm{RR}}(-1)^{\mathrm{J}_{\mathrm{L}}} \mathrm{J}_{\mathrm{L}}(-1)^{\mathrm{J}_{\mathrm{R}}} \mathrm{J}_{\mathrm{R}} \mathrm{q}^{\mathrm{H}} \overline{\mathrm{q}}^{\tilde{\mathrm{H}}}-\text { const }\right] \\
& =F_{1}^{c p l x}+F_{1}^{\text {kahler }}
\end{aligned}
$$

Here the trace is over the Ramond-Ramond sector and $J_{L}, J_{R}$ are the left and right-moving $U(1)$ currents of the $(2,2)$ superconformal algebra. As shown in [8], $F_{1}$ splits as a sum that depend only on complex and Kahler moduli respectively. These terms are exchanged by mirror symmetry. 
The formula analogous to (2.19) was given in [8]:

$$
\begin{aligned}
F_{1}^{I I} & =\log \left[e^{\left(3+h_{1,1}-\chi / 12\right) K}\left(\operatorname{det} K_{i \bar{j}}\right)^{-1}\left|\frac{1}{\Psi_{1}^{I I}(t)}\right|^{2}\right] \\
& =\left(3+h_{1,1}-\frac{\chi}{12}\right) K-\log \operatorname{det} K_{i \bar{j}}-2 \log \left|\Psi_{1}^{I I}(t)\right| \\
& \equiv-\log \left\|\Psi_{\text {grav }}^{I I}(t)\right\|^{2}
\end{aligned}
$$

The type II result analogous to (2.10) was obtained in [17]. Massless particles lead to a singularity in $F_{1}$ of:

$$
\frac{1}{6}\left[\sum_{v m} \log m^{2}-\sum_{h m} \log m^{2}\right]
$$

In order to go further we must specialize to Calabi-Yau spaces $X_{3}$ which have heterotic duals. This requires that $X_{3}$ be a K3 fibration [18, 19].

\subsection{Application to K3 fibrations}

We now assume $X_{3}$ is a $K 3$ fibration and follow the discussion of Aspinwall and Louis [19]. We choose integral generators $e_{s}, e_{a}$ of $H^{2}\left(X_{3} ; Z\right)$ and introduce coordinates $\left(t_{s}, t_{a}\right)$ on the complexified Kahler form:

$$
\omega=t_{s} e_{s}+\sum_{a=1}^{s+2} t_{a} e_{a}
$$

where $e_{s}$ is dual to the K3 fiber. The $e_{a}$ form a basis for integral $(1,1)$ classes for the K3 fiber. The Picard lattice Pic $(K 3)$ will have signature $(1, s+1)$ and the $t_{\alpha}$ are coordinates for the Kahler cone in $\operatorname{Pic}(K 3) \otimes \mathbb{C}$. Therefore, we may write:

$$
\begin{aligned}
n_{V} & =s+4 \\
3+h_{1,1}-\chi / 12 & =\left(12+5 n_{V}+n_{H}\right) / 6
\end{aligned}
$$

The coordinates $t_{s}, t_{a}$ are flat (special) coordinates and in [19] it is shown that we have the identification:

$$
\begin{aligned}
t_{s} & =4 \pi i S \\
t_{a} & =y^{a}
\end{aligned}
$$

The holomorphic anomaly at large $\operatorname{Im}\left(t_{s}\right)$ is computed from

$$
\begin{aligned}
K & =K^{(0)}+\mathcal{O}\left(1 / \operatorname{Im}\left(t_{s}\right)\right) \\
K^{(0)} & =-\log \left[\operatorname{Im}\left(t_{s}\right)\right]-\log \left[-\left(\operatorname{Im} t_{\alpha}\right)^{2}\right]+\text { const. }
\end{aligned}
$$


giving:

$$
\begin{aligned}
F_{1}^{I I} & =-\frac{5 n_{V}+n_{H}}{6} \log [\operatorname{Re} S]+\frac{4 \pi^{2}}{3} 24 \operatorname{Re}(S) \\
& +\frac{n_{V}-n_{H}-24}{6} \log \left[-2\left(\operatorname{Imt}_{\alpha}\right)^{2}\right] \\
& -\log \left|\Psi^{I I}\left(t_{\alpha}\right)\right|^{2}+\mathcal{O}\left(e^{2 \pi i t_{s}}\right)
\end{aligned}
$$

The fact that there are only exponential corrections is surprising since we expect $K, \log \operatorname{det} K_{i \bar{j}}$ to be corrected to all orders in $1 / \operatorname{Im}\left(t_{s}\right)$. However, it is implied by (2.20).

We conclude that at large $\operatorname{Re}(S)$ we have:

$$
\Psi_{\text {grav }}^{I I}\left(t_{s}, t_{a}\right)=q_{\tilde{S}}^{2} \Psi_{\text {grav }}^{I I}\left(t_{a}\right)\left(1+\mathcal{O}\left(q_{S}\right)\right)
$$

Now, $F_{1}^{I I}$ must be an invariant function on the moduli space. Combined with (3.4) we obtain two conditions which are the analogs of the heterotic conditions (2.16) and (2.18):

1. $\Psi_{\text {grav }}^{I I}\left(t_{\alpha}\right)$ is a modular form of weight

$$
\frac{1}{12} b_{\text {grav }}=4-\chi / 12
$$

2. The zeroes of $\Psi_{\text {grav }}^{I I}\left(t_{a}\right)$ at an enhanced symmetry point with hypermultiplet and vectormultiplet masses vanishing to first order are of order:

$$
\frac{1}{6}\left[\Delta n_{H}-\Delta n_{V}\right]
$$

when $\tilde{S}$ is well-defined.

Now we can apply the Koecher principle: Modular forms on $\mathcal{N}^{s+2,2}$ are completely determined by their weight, zeroes and poles. In general we need to allow for phases (i.e., a "multiplier system") in the modular transformation laws. However, such phases correspond to one-dimensional representations of the T-duality group $O(\Gamma)$. It follows from a theorem of Kazhdan [20] that the abelianization $O(\Gamma) /[O(\Gamma), O(\Gamma)]$ is always a finite group, and therefore, we can eliminate the phases by raising the form to an appropriate power. $\mathrm{O}$ Comparing (3.11) (3.12) with (2.17) and (2.18) we learn that (up to a phase):

$$
\Psi_{\text {grav }}^{H E T}\left(y^{a}\right)=\Psi_{\text {grav }}^{I I}\left(t_{\alpha}\right)
$$

on very general grounds.

3 For the example considered in the next section the power 4 is sufficient. 


\section{Gravitational correction for the FHSV model}

Computation of the integral expression (2.5) leads to interesting automorphic forms associated to threshold corrections. In this section we work in reverse: we use a known result on automorphic forms to determine a threshold correction.

We will consider the FHSV model introduced in [4]. A discussion of phase transitions in this model and a careful discussion of some topological subtleties in its definition can be found in [21]. The model consists of an $N=2, d=4$ string dual pair where the type IIA theory is formulated on a Calabi-Yau manifold of the form $X_{3}=\left(T^{2} \times S\right) / \mathbb{Z}_{2}$ where $S$ is a $K 3$ surface which double covers an Enriques surface. The $\mathbb{Z}_{2}$ acts as $z \rightarrow-z$ on $T^{2}$ and as the free Enriques involution on $S$. In addition there are some discrete degrees of freedom which must be included [4:21]. The resulting Calabi-Yau space has Betti numbers $h^{1,1}\left(X_{3}\right)=h^{2,1}\left(X_{3}\right)=11$ and hence $\chi\left(X_{3}\right)=0$.

An important property of this model is that the exact vectormultiplet moduli space is a quotient of:

$$
S L(2, \mathbb{R}) / S O(2) \times O(10,2) /[O(10) \times O(2)]
$$

with its natural Kähler metric. That is, the special Kähler geometry of the moduli space (4.1) does not receive string quantum corrections because of the decoupling of vector and hypermultiplets and it was argued in [1] that it is also uncorrected by string world-sheet quantum corrections from world-sheet instantons. It thus follows that $\mathcal{F} \sim S y^{2}$ and $K=-\log [R e S]-\log \left[-(\operatorname{Im} y)^{2}\right]$ exactly. Nevertheless, there will be nontrivial quantum corrections for other $F$-terms in the low energy effective theory. The relevant automorphic form for the $R^{2}$ coupling is described in the next section.

The global identifications are associated with duality transformations, and can be determined from the lattice of RR charges. It was shown in [⿴囗十,21] that the moduli space is:

$$
\mathcal{M}_{\mathrm{vm}}^{\text {quantum }}=S L(2, \mathbb{Z}) \backslash S L(2, \mathbb{R}) / S O(2) \times O(M) \backslash O(10,2) /[O(10) \times O(2)] .
$$

Here $O(M)$ is the group of automorphisms of

$$
M \equiv E_{8}(-2) \oplus I I^{1,1}(2) \oplus I I^{1,1}(1)
$$

(this is the lattice denoted $M$ in [22]). Here $I I^{1,1}$ is the even self-dual Lorentzian lattice and for any self-dual lattice $L, L(n)$ denotes the lattice with norm-squared rescaled by $n$. (4 $M$ may be interpreted in many ways. One way is that it is the sublattice of integral cohomology $H^{*}(S ; \mathbb{Z})$ even under the Enriques involution.

4 The sign convention in (4.3) is chosen to agree with the standard convention of algebraic geometry 


\subsection{Determining $F_{1}$}

In order to determine $F_{1}$ we first use the representation (3.2) from the type II side. Note first that the dependence on the Kahler moduli of $T^{2}$ can only come from the untwisted unprojected sector of the $\mathbb{Z}_{2}$ orbifold. The calculation in the untwisted sector is, up to a factor of $1 / 2$, the same as the calculation on a Calabi-Yau manifold of the form $T^{2} \times S$ where $S$ is a K3 surface. For the latter manifold one easily finds

$$
F_{1}^{\text {kahler }}=-24 \log \left\|\eta^{2}(T)\right\|^{2}
$$

where $T$ is the Kahler modulus of $T^{2}$, the factor of 24 comes from the elliptic genus of $\mathrm{K} 3$, and the T-duality invariant norm is $\left\|\eta^{2}(T)\right\|^{2} \equiv \operatorname{Im} T\left|\eta^{2}(T)\right|^{2}$. To compare this to the discussion in sec. 3.1 we view this as a $K 3$ fibration where the $\mathbb{P}^{1}$ base is $T^{2} / \mathbb{Z}_{2}$. Since the area of this $\mathbb{P}^{1}$ is half that of $T^{2}$ we should identify $t_{s}=T / 2$. Taking into account this identification and the factor of two from the orbifold we have:

$$
F_{1}=\log \left\|\frac{1}{\eta^{24}\left(2 t_{s}\right) \Psi^{I I}}\right\|^{2}
$$

where $\Psi^{I I}$ is a section of a line bundle over $O(M) \backslash O(10,2) /[O(10) \times O(2)]$, or, equivalently, an automorphic form for $O(M)$. We will now use our knowledge of the physics to determine the weight of the form and its singularities.

The weight is easily determined from (3.11) to be $w=4-\chi / 12=4$. The singularities of $\Psi^{I I}$ must come from singular Calabi-Yau manifolds $X_{3}$. As explained in [21] $X_{3}$ becomes singular when

1. $S$ develops an ADE singularity, or

2. $S$ develops a quantum singularity, which by mirror symmetry can be viewed as a singularity occuring at points in the moduli space where the $\mathbb{Z}_{2}$ fails to act freely. As explained in [21, the singularities of the second kind occur at the divisor in $O(10,2) / O(10) \times O(2)$ orthogonal to a norm -2 vector. 6

Physically, the singularities of the first kind correspond to theories with enhanced $N=4$ supersymmetry. At these points we have $\Delta n_{H}-\Delta n_{V}=0$. There is thus no singularity in $\Psi$ at such points. On the other hand, singularities of the second kind

5 The physical implications of this are explored in 23.

6 This is the divisor denoted $\cup H_{d}$ in 22]. As shown in that paper, there is only one $O(M)$ orbit. 
correspond to enhanced $N=2, S U(2), N_{f}=4$ theories. Using (3.12) we see that along this divisor $\Psi$ will have a zero of order

$$
\frac{1}{6}[8-2]=1
$$

The above properties of $\Psi$ are also easily reproduced within the dual heterotic theory. The heterotic dual is obtained as an asymmetric $\mathbb{Z}_{2}$ orbifold for lattices in $\mathbb{R}^{22,6}$ with orthogonal decomposition:

$$
\Gamma^{9,1} \oplus \Gamma^{9,1} \oplus \Gamma^{1,1} \oplus \Gamma^{1,1} \oplus \Gamma^{2,2}
$$

where the $\mathbb{Z}_{2}$ acts as $\left|P_{1}, P_{2}, P_{3}, P_{4}, P_{5}\right\rangle \rightarrow e^{2 \pi i \delta \cdot P_{3}}\left|P_{2}, P_{1}, P_{3},-P_{4},-P_{5}\right\rangle$ with $\delta$ the order two shift vector defined in [4]. The weight is now determined from (2.16) and the enhanced symmetry divisors correspond to the $N=4$ and $N=2$ theories described above [4]. Since the vector multiplet moduli space is uncorrected there is no possible singularity in $\tilde{S}=S$, and we recover the above result on the zeros of $\Psi_{\text {grav }}^{H E T}$.

In principle the correction $F_{1}$ could be obtained by evaluating (2.5) explicitly as in [1,13, 14, 15]. However, this is not necessary since enough is known about the relevant modular forms to determine $F_{1}$ without calculation. As we have mentioned, by the Koecher principle, a nonsingular modular form for $O(M)$ is determined by its weight and the order of its zeros on $O(10,2) /[O(10) \times O(2)]$. The reason is that two forms of the same weight and zeroes would have a ratio which is an $O(M)$-invariant holomorphic function $\Psi_{12}=\Psi_{1} / \Psi_{2}$ on $O(10,2) /[O(10) \times O(2)]$. This would descend to the quotient space $O(M) \backslash O(10,2) /[O(10) \times O(2)]$. However, this space has a compactification (the "BailyBorel compactification") by adding varieties of dimension zero and one [24] [25]. Hence, by Hartog's theorem $\Psi_{12}$ must be a constant.

It turns out that an automorphic form of precisely the required weight and with precisely the required zeros has already been constructed by Borcherds in [22]. We call this form $\Phi_{B E}$, the Borcherds-Enriques form. It may be defined as follows.

We first make a choice of null vector $v \in M \cong\left[\Gamma^{9,1} \oplus \Gamma^{9,1}\right]^{\mathbb{Z}_{2}} \oplus I I^{1,1}(1)$. 7 Here the $\mathbb{Z}_{2}$ superscript indicates that we take the part of the lattice invariant under the exchange of the two factors. We next define $L=\left(v^{\perp} / v \mathbb{Z}\right)$. In particular, we make the choice of a primitive null vector in $I I^{1,1}(2)$ so that $L \cong E_{8}(-2) \oplus \Gamma^{1,1}(1)$. Let $r>0$ denote lattice

7 This choice of null vector corresponds to a choice of which integers we use for Poisson resummation in the calculation of [1]. 
vectors in the forward light-cone. Denoting vectors in the lattice by $r=(\vec{b} ; m, n) \in L$ and points in a tube domain by $y \in \mathbb{R}^{1,9}+i C^{+}$we define [22]:

$$
\begin{aligned}
\Phi_{B E}(y) & \equiv e^{2 \pi i \rho \cdot y} \prod_{r>0}\left(1-e^{2 \pi i r \cdot y}\right)^{(-1)^{m+n}} d\left(-r^{2} / 2\right) \\
\sum d(n) q^{n} & =q^{-1} \frac{\prod\left(1+q^{2 n+1}\right)^{8}}{\prod\left(1-q^{2 n}\right)^{8}}
\end{aligned}
$$

where $\rho=(0 ; 0,1)$ is a Weyl vector. It is shown in [22] that the form $\Phi_{B E}(y)$ satisfies two key properties:

1. $\Phi_{B E}(y)$ is an automorphic form on $O(10,2) /[O(10) \times O(2)]$ for the discrete group $O(M)$ of weight 4 .

2. The zeroes of $\Phi_{B E}(y)$ are first order and are located on the divisor of $O(10,2) / O(10) \times$ $O(2)$ of negative definite subspaces orthogonal to norm -2 vectors in $M$.

As we have remarked several times, these two properties determine $\Phi$ up to a constant, so we can finally obtain the full nonperturbative result for the gravitational threshold correction:

$$
F_{1}=\log \left\|\frac{1}{\eta^{24}\left(2 \tau_{S}\right) \Phi_{B E}(y)}\right\|^{2}
$$

where we have written the final answer in terms of the heterotic dilaton $\tau_{S}$.

The factor of $2 \tau_{S}$ in (4.9) has physical significance and is quite closely related to a similar factor in the discussion of $N=2$ gauge theory with matter given in [26]. Expanding (4.9) gives a term linear in $S$ and a power series in $q_{S}^{2}$. The latter should be interpreted as coming from zero size instantons as in [23]. Since a single instanton has action $q_{S}$ this indicates that only even numbers of instantons contribute to $F_{1}$. A similar result was found in [26] for $N=2$ theories with matter as a result of an anomalous $\mathbb{Z}_{2}$ symmetry identified with the center of the global $O\left(2 N_{f}\right)$ flavor group. In that situation amplitudes with odd numbers of instantons were odd under the $\mathbb{Z}_{2}$ and thus did not contribute to the even metric on moduli space. In our situation there is also a natural $\mathbb{Z}_{2}$, it is the $\mathbb{Z}_{2}$ which is +1 on all untwisted states of the $\mathbb{Z}_{2}$ orbifold and -1 on all twisted states. The above result tells us that again odd numbers of instantons are odd under this $\mathbb{Z}_{2}$ and hence do not contribute to $F_{1}$ which is even. Note also that at enhanced symmetry points with $N=2$ supersymmetry and gauge group $S U(2)$ with $N_{f}=4$ the four matter multiplets arise from the twisted sector of the orbifold and thus are odd under the $\mathbb{Z}_{2}$. The orbifold $\mathbb{Z}_{2}$ thus agrees with the $\mathbb{Z}_{2}$ of [26] when restricted to the $N=2$ gauge sector. 


\section{Application 1: K3 determinants}

The dependence of $F_{1}$ on complex structures was determined in [8] to be given by a combination of Ray-Singer torsions. Specifically we have:

$$
F_{1}^{\text {complex }}=+\sum_{0 \leq p, q \leq 3} p q(-1)^{p+q} \log \operatorname{Det}^{\prime} \Delta_{\bar{\partial}}^{p, q}
$$

We have determined this quantity for $X_{3}=\left(T^{2} \times S\right) / \mathbb{Z}_{2}$. Using special properties of this manifold we can extract a relation between $\Phi_{B E}$ and determinants of $\bar{\partial}$ operators on K3 surfaces. Since $X_{3}$ is a self-mirror family we can work equally well with Kahler or complex moduli, a fact which will be exploited in the next section.

Using the Hodge dual we obtain an isospectral isomorphism $\Omega^{p, q} \cong \Omega^{q, p}$ and hence: $\operatorname{Det}^{\prime} \Delta_{\bar{\partial}}^{p, q}=\operatorname{Det}^{\prime} \Delta_{\bar{\partial}}^{q, p}$. Moreover, using the covariantly constant 3 -form we have a second isospectral isomorphism: $\Omega^{0, q} \cong \Omega^{3, q}$. Finally, let $\Omega_{\perp}^{1, q}$ denote the space of forms orthogonal to the harmonic forms. We can obtain the isospectral isomorphism:

$$
\begin{aligned}
\Omega_{\perp}^{1, q} & \cong \bar{\partial}\left(\Omega^{0, q}\right) \oplus \bar{\partial}^{\dagger}\left(\Omega^{2, q}\right) \\
& \cong \bar{\partial}^{\dagger}\left(\Omega^{3, q}\right) \oplus \bar{\partial}\left(\Omega^{1, q}\right) \cong \Omega_{\perp}^{2, q}
\end{aligned}
$$

and hence $\operatorname{Det}^{\prime} \Delta_{\bar{\partial}}^{p, q}=\operatorname{Det}^{\prime} \Delta_{\bar{\partial}}^{3-p, q}$. It follows that:

$$
F_{1}=9 \log \operatorname{Det}^{\prime} \Delta^{0,0}-6 \log \operatorname{Det}^{\prime} \Delta^{1,0}+\log \operatorname{Det}^{\prime} \Delta^{1,1} .
$$

Moreover, the metric is a product on $T^{2} \times S$ where $T^{2}$ is the torus and $S$ is the K3 surface which double-covers the Enriques surface. Thus, $\Delta=\Delta_{T^{2}}+\Delta_{S}$ and the Laplacian is block diagonal if we decompose the space of $(p, q)$ forms according to:

$$
\Omega^{p, q}\left(\left(T^{2} \times S\right)\right) \cong \oplus_{p^{\prime}, q^{\prime}} \Omega^{p-p^{\prime}, q-q^{\prime}}\left(T^{2}\right) \otimes \Omega^{p^{\prime}, q^{\prime}}(S)
$$

Also, the involution $\left(-1, I_{\text {Enriques }}\right)$ on $T^{2} \times S$ commutes with the Laplacian so we can decompose all spaces into the \pm eigenspaces under the involution.

We also need some facts about the spectrum of the Laplacians $\Delta_{S}^{p, q}$ and their determinants which we define using zeta-function regularization. The spectrum of the Laplacian on 0 -forms leads to a zeta-function which can naturally be written as a sum of zeta functions for the even and odd functions on $S$ :

$$
\zeta_{S}(s)=\zeta_{S}^{+}(s)+\zeta_{S}^{-}(s)
$$


All the other relevant $\zeta$ functions for $S$ can be written in terms of $\zeta_{S}^{ \pm}$. Using the fact that the covariantly constant $(2,0)$ form on $S$ is odd one can derive the relevant zeta functions for the Laplacian acting on other forms. In particular:

$$
\begin{array}{ll}
\Omega_{ \pm}^{1,0}: & \zeta_{S}^{+}(s)+\zeta_{S}^{-}(s) \\
\Omega_{ \pm}^{1,1}: & 2 \zeta_{S}^{+}(s)+2 \zeta_{S}^{-}(s)
\end{array}
$$

We now consider the $\zeta$-function on $\Omega_{ \pm}^{p-p^{\prime}, q-q^{\prime}}(S) \otimes \Omega_{ \pm}^{p^{\prime}, q^{\prime}}\left(T^{2}\right)$. The spectrum of the Laplacian is the sum of pairs $\lambda_{i}^{ \pm}(S)+\lambda_{j}^{ \pm}\left(T^{2}\right)$. In fact, we know the spectrum $\lambda_{j}\left(T^{2}\right)$ of the flat torus quite explicitly, so the $\zeta$-function is of the form

$$
\sum_{i, n_{1}, n_{2}}\left(\lambda_{i}^{ \pm}(S)+\frac{\left|n_{1} \tau+n_{2}\right|^{2}}{\tau_{2}^{2}}\right)^{-s}
$$

It is quite important to separate out the terms corresponding to a zeromode on $T^{2}$ or $S$ from expressions such as (5.7). Let us define the functions

$$
\begin{aligned}
& \mu^{+}(s)=\sum_{\lambda_{i}^{+} \neq 0,\left(n_{1}, n_{2}\right) \neq 0}\left(\lambda_{i}^{+}(S)+\frac{\left|n_{1} \tau+n_{2}\right|^{2}}{\tau_{2}^{2}}\right)^{-s} \\
& \mu^{-}(s)=\sum_{\lambda_{i}^{-} \neq 0,\left(n_{1}, n_{2}\right) \neq 0}\left(\lambda_{i}^{+}(S)+\frac{\left|n_{1} \tau+n_{2}\right|^{2}}{\tau_{2}^{2}}\right)^{-s}
\end{aligned}
$$

Then, a short calculation produces the following $\zeta$-functions

$$
\begin{array}{ll}
\Omega_{+}^{0,0}\left(X_{3}\right) & \zeta_{T^{2}}^{+}(s)+\zeta_{S}^{+}(s)+\mu^{+}+\mu^{-} \\
\Omega_{+}^{1,0}\left(X_{3}\right) & \zeta_{T^{2}}^{-}(s)+\zeta_{S}^{+}(s)+2 \zeta_{S}^{-}(s)+3 \mu^{+}+3 \mu^{-} \\
\Omega_{+}^{1,1}\left(X_{3}\right) & \left(n_{e}+n_{0}+1\right) \zeta_{T^{2}}^{-}(s)+5 \zeta_{S}^{+}(s)+4 \zeta_{S}^{-}(s)+9 \mu^{+}+9 \mu^{-}
\end{array}
$$

where $n_{e}, n_{0}$ are the dimensions of the even and odd harmonic (1,1)-forms. For our example these are $n_{e}=n_{0}=10$. Computing the combination in (5.3) we find that the mixed eigenvalues in $\mu^{ \pm}$cancel out and we are left with

$$
24 \zeta_{T^{2}}^{+}+8\left(\zeta_{S}^{+}-\zeta_{S}^{-}\right)
$$

and we recover the fact that $F_{1}$ can be written as a product of the function for $T^{2}$ and a function for $S$. Moreover, we obtain the result

$$
8\left[\log \operatorname{Det}^{\prime} \Delta_{+}^{0,0}-\log \operatorname{Det}^{\prime} \Delta_{-}^{0,0}\right]=-\log \left\|\Phi_{B E}\right\|^{2}
$$

This result is closely related to a result of Jorgenson and Todorov [27. 


\section{Application 2: Mirror maps and curve counting}

In $F$-compactification to 8 dimensions the Narain moduli space $\mathcal{N}^{2,18}$ plays an important role [28]. On the heterotic side it is natural to describe this space in terms of tube domain coordinates as $t^{i} \in \mathbb{R}^{1,17}+i C^{+}$. On the F-theory side one parametrizes the space in terms of the positions $z_{i} \in \mathbb{P}^{1}$ where 7-branes have been inserted. Equivalently the elliptic fiber of the elliptically fibered $K 3$ degenerates at points $z_{i}$. It would be very interesting to determine the exact mapping between the $z_{i}$ and the $t^{i}$. If we restrict to the $O(10,2)$ subspace corresponding to Enriques double-covers (all of which are elliptically fibered) then this is simply the mirror map. The above results give some nontrivial information on this map. (See [29] for an explicit determination of the map on a two-parameter subspace.)

The proper interpretation of (3.3) is that $\Psi_{\text {grav }}^{I I}(t)$ is a section of bundle with a hermitian metric. Using special coordinates and the mirror map we can define a trivialization near a large Kähler structure boundary point in terms of the fundamental holomorphic period $\varpi_{0}$. Then if $t(z)$ is the mirror map:

$$
\left(\varpi_{0}\right)^{3+h_{1,1}-\chi / 12} \operatorname{det}(\partial t / \partial z) \Psi_{\text {grav }}(t)
$$

is an invariant object: it is the quotient of two sections. On the other hand, we know that its only singularities can occur on the discriminant locus. Hence, in the case of the Enriques surfaces, we expect (6.1) with $\Psi_{\text {grav }}(t)=\Phi_{B E}(t)$ to be proportional to $\prod_{i j}\left(z_{i}-z_{j}\right)^{n_{i j}}$ for some integers $n_{i j}$. The integers $n_{i j}$ can be determined by counting elliptic curves near the discriminant locus. Indeed, once $t(z)$ is known one could then proceed to count elliptic curves in the Calabi-Yau $X_{3}$ (and hence extract elliptic curves in the Enriques surface) using the result [8] [16]:

$$
\begin{aligned}
-\log \left[\left(\varpi_{0}\right)^{3+h_{1,1}-\chi / 12} \operatorname{det}(\partial t / \partial z) \Psi_{\text {grav }}(t)\right] & =-\frac{4 \pi i}{12} \int_{X_{3}} c_{2} \wedge \omega \\
-2 & \sum_{\Sigma \in H_{2}\left(X_{3} ; \mathbb{Z}\right)} n^{(1)}(\Sigma) \log \left[\eta\left(e^{2 \pi i \int_{\Sigma} \omega}\right)\right]-\frac{1}{6} \sum_{\Sigma \in H_{2}\left(X_{3} ; \mathbb{Z}\right)} n^{(0)}(\Sigma) \log \left[1-e^{2 \pi i \int_{\Sigma} \omega}\right]
\end{aligned}
$$

where $n^{(1)}, n^{(0)}$ are the number of elliptic and rational instantons and $\omega$ is the complexified Kähler class. Since these are not isolated the counting of these instantons is subject to the standard caveats. 


\section{Conclusions and Conjectures}

The main motivation for this paper was the hope that the present result will lead to a better understanding of the relation between the BPS algebras discussed in [2] and threshold corrections. Given the result (4.5) we are led to the following conjecture.

Conjecture: For heterotic compactification on $K 3 \times T^{2}$ we always have

$$
F_{1}=\log \left\|\frac{1}{q_{\tilde{S}}^{2} \Phi(y)}\left(1+\mathcal{O}\left(q_{S}\right)\right)\right\|^{2}
$$

where $\Phi(y)$ is the denominator product for a GKM algebra closely related to the algebra of perturbative BPS states.

Understanding the precise relation between the algebra associated with the denominator product and the algebra of BPS states remains elusive. In the FHSV model considered here $\Phi(y)$ is the denominator product for the "fake monster Lie superalgebra" [3]. (It can also be interpreted as the trace of a involution in the Monster in the Lie algebra cohomology of the Monster Lie algebra.) The algebra of BPS states in the FHSV model resembles a generalized Kac-Moody algebra, but is graded by $\Gamma^{10,2}$. Our result strongly suggests that some subquotient of the algebra of BPS states, perhaps associated to cohomology associated with a null direction, will be the "fake monster super Lie algebra." It would be very interesting to make this suggestion concrete, but we have not done so. In this connection it is intriguing to note that by expanding the same form around different cusps one can obtain distinct GKM's [30].

On a more speculative note, it is tempting to conjecture that (7.1) is the first term in an expansion in powers of $q_{S}$ of an automorphic form which governs the exact, nonperturbative gravitational threshold correction in all $N=2$ theories: $F_{1}=-\log \|\Phi\|^{2}$, and that $\Phi$ is associated to an electric or magnetic subalgebra of the full algebra of BPS states associated to the Calabi-Yau space [2].

Finally, an interesting direction for future research is to understand if there is a connection between BPS algebras and higher dimensional current algebras. There is a hint of such a connection since the quantity $F_{1}$ also appears in [31.

\section{Acknowledgements}

We would like to thank R. Borcherds, M. Douglas, A. Gerasimov, J. Jorgenson, A. Losev, D. Morrison, N. Nekrasov, S. Shatashvili, A. Strominger, A. Todorov, and G. 
Zuckerman for discussions. We are extremely grateful to J. Louis for extensive correspondence on gravitational couplings and holomorphic anomalies, and for comments on the manuscript. We are also grateful to V. Kaplunovsky for an important discussion on this topic.

GM would like to thank the Aspen Center for Physics for providing a stimulating atmosphere during the beginning of this work. This work was supported in part by NSF Grant No. PHY 91-23780 and DOE grant DE-FG02-92ER40704. 


\section{References}

[1] J. A. Harvey and G. Moore, "Algebras, BPS states, and strings," Nucl. Phys. B463(1996)315; hep-th/9510182.

[2] J. A. Harvey and G. Moore, "On the algebra of BPS states," hep-th/9609017.

[3] R. E. Borcherds, "Monstrous moonshine and monstrous Lie superalgebras," Invent. Math. 109 (1992) 405.

[4] S. Ferrara, J. A. Harvey, A. Strominger, C. Vafa , "Second-Quantized Mirror Symmetry," Phys. Lett. B361 (1995) 59; hep-th/9505162.

[5] I. Antoniadis, E. Gava, K.S. Narain, "Moduli corrections to gravitational couplings from string loops," Phys. Lett. B283 (1992) 209, hep-th/9203071; " Moduli corrections to gauge and gravitational couplings in four-dimensional superstrings," Nucl. Phys. B383 (1992) 109, hep-th/9204030.

[6] S. Cecotti, S. Ferrara, L. Girardello, A. Pasquinucci, M. Porrati, "Matter coupled supergravity with Gauss-Bonnet invariants: Component Lagrangian and supersymmetry breaking," Int. J. Mod. Phys. A3 (1988) 1675.

[7] G.L. Cardoso, D. Lust, and B.A. Ovrut, "Moduli dependent non-holomorphic contributions of massive states to gravitational couplings and $C^{2}$ terms in $Z(N)$ orbifold compactification," Nucl. Phys. B436 (1995) 65; hep-th/9410056.

[8] M. Bershadsky, S. Cecotti, H. Ooguri and C. Vafa, "Kodaira-Spencer theory of gravity and exact results for quantum string amplitudes, " Commun. Math. Phys. 165 (1994) 311, hep-th/9309140.

[9] V. Kaplunovsky, J. Louis and S. Theisen, "Aspects of duality in $N=2$ string vacua," Phys. Lett. B357 (1995) 71, hep-th/9506110.

[10] G. L. Cardoso, G. Curio, D. Lust, T. Mohaupt, S.-J. Rey, "BPS Spectra and NonPerturbative Couplings in N=2,4 Supersymmetric String Theories," Nucl. Phys. B464 (1996) 18; hep-th/9512129.

[11] E. Kiritsis, C. Kounnas, P.M. Petropoulos, J. Rizos, hep-th/9605011

[12] L. Dixon, V. S. Kaplunovsky and J. Louis, "Moduli-dependence of string loop corrections to gauge coupling constants, "Nucl. Phys. B329 (1990) 27.

[13] Toshiya Kawai , "String Duality and Modular Forms," hep-th/9607078.

[14] M. Henningson and G. Moore, "Threshold Corrections in $K 3 \times T 2$ Heterotic String Compactifications," hep-th/9608145.

[15] G. L. Cardoso, G. Curio, D. Lust, "Perturbative Couplings and Modular Forms in $\mathrm{N}=2$ String Models with a Wilson Line," hep-th/9608154.

[16] M. Bershadsky, S. Cecotti, H. Ooguri and C. Vafa, "Holomorphic anomalies in topolgical field theories," Nucl. Phys. B405 (1993) 279; hep-th/9302103.

[17] C. Vafa, "A stringy test of the fate of the conifold," Nucl. Phys. B447 (1995) 252; hep-th/9505023. 
[18] A. Klemm, W. Lerche and P. Mayr, "K3-fibrations and Heterotic-Type II string duality, " Phys. Lett. B357 (1995) 313, hep-th/9506122.

[19] P. Aspinwall and J. Louis, "On the Ubiquity of K3 fibrations in in string duality," Phys. Lett. B369 (1996) 233; hep-th/9510234.

[20] C. Delaroche and A. Kirillov, "Sur les relations entre l'espace dual d'un groupe et la structure de ses sous-groupes fermés," Sem. Bourbaki, 1968, p. 343.

[21] P. Aspinwall, "An $N=2$ dual pair and a phase transition," Nucl. Phys. B460 (1996) 57 ; hep-th/9510142.

[22] R. E. Borcherds, "The moduli space of Enriques surfaces and the fake monster Lie superalgebra," Topology vol. 35 no. 3, (1996) 699.

[23] J. A. Harvey and G. Moore, "Five-brane instantons and $R^{2}$ couplings in $N=4$ string theory," hep-th/9610237.

[24] H. Sterk, "Compactifications of the period space of Enriques surfaces. Part I," Math. Z. $207(1991) 1$

[25] F. Scattone, On the compactification of moduli spaces for algebraic K3 surfaces, Mem. of the AMS, 374 (1987)

[26] N. Seiberg and E. Witten, "Monopoles, duality and chiral symmetry breaking in $N=2$ supersymmetric QCD, " Nucl. Phys. B431 (1994) 484, hep-th/9408099.

[27] J. Jorgenson and A. Todorov, "Enriques surfaces, analytic discriminants, and Borcherd's $\Phi$ function," Yale preprint.

[28] C . Vafa, "Evidence for F-Theory," hep-th/9602022; D. R. Morrison, C. Vafa, "Compactifications of F-Theory on Calabi-Yau Threefolds - I," hep-th/9602114; D. R. Morrison, C. Vafa, "Compactifications of F-Theory on Calabi-Yau Threefolds - II," hep-th/9603161.

[29] G. L. Cardoso, G. Curio, Dieter Lust, T. Mohaupt, "On the Duality between the Heterotic String and F-Theory in 8 Dimensions," hep-th/9609111

[30] R. Borcherds, "Automorphic forms with singularities on Grassmannians," alg-geom/9609022.

[31] A. Losev, G. Moore, N. Nekrasov, and S. Shatashvili, "Chiral Lagrangians, Anomalies, Supersymmetry, and Holomorphy," hep-th/9606082. 